\title{
Determination of Small-Amount Polypropylene in Imported Recycled Polyethylene/Polypropylene Blends by Fourier Transform Infrared Spectroscopy
}

\author{
ShujunDai ${ }^{1}$, ChuanLuo ${ }^{2}$, ChaoZhang ${ }^{3}$, HaoWang $^{2}$, YinZhang ${ }^{3}$ and LifengYuan ${ }^{2 *}$ \\ ${ }^{1}$ Ningbo Cross-border E-commerce Promotion Center, LinQiao Road 190\#, NingBo, 315012, P. R. China \\ 2 Ningbo Customs District, QinYi Road 66\#, NingBo, 315012, P. R. China \\ ${ }^{3}$ Ningbo academy of science and technology for inspection and quarantine, QinYi Road 66\#, NingBo, 315012, P. R. China
}

\begin{abstract}
In the domestic production of recycled plastics, it is difficult to separate olefin polymers such as polyethylene and polypropylene. Therefore, the prerequisite for identifying the cargo-solid waste properties of recycled plastics is to determine the polymer composition. In this paper, Fourier transform infrared spectroscopy was used to detect polypropylene content in polyethylene and polypropylene blends. Under $2 \%$ $8 \%$ polypropylene content, an exponential relationship was shown in the ratio of characteristic peak area. This method features fast detection speed and accurate judgment. It can quickly determine whether the polypropylene content in the polyethylene and polypropylene blends is greater than $5 \%$ in the tariff inspection. Also, it can determine plastic solid waste attributes and identify whether polyethylene is mixed with a small amount of polypropylene content.
\end{abstract}

\section{Introduction}

In the early stage of this century, China imported a large amount of waste plastics to supplement the shortage of raw materials. Recently, due to changes in domestic environmental protection and development policies, the import of waste plastics has been banned. A new phenomenon of recycled plastic pellets imported thus appear, with import volume increased significantly. However, recycled plastic pellets have unknown cargosolid waste properties and lack criteria for determination. The situation has caused a backlog of cargo in the port, which incurs companies' intense response. Therefore, research in this area is urgently needed.

Fourier transform infrared spectroscopy features high detection sensitivity, high measurement accuracy, high resolution, fast measurement speed, low astigmatism and wide band. Infrared spectroscopy is an effective means to study and characterize molecular structure. The position, intensity, and shape of characteristic absorption peaks can be used to qualitatively characterize the polymer content ${ }^{[1,2]}$. For attribute identification of recycled plastic solid waste, we often use FT-IR to determine the main sample components, but the conventional DSC method has big deviations for low-content polymers [3-5], and other pyrolysis gas chromatography/ mass spectroscopy methods cannot identify polyethylene and polypropylene [6-8], though both are olefin polymers. It is therefore necessary to develop an infrared spectroscopy method able to quickly identify the composition of olefin polymers.

There are many papers on polyethylene and polypropylene blends at home and abroad, but all focus on characterization of new materials, with few researches on recycled materials. Determination of polyethylene and polypropylene blends is mainly based on SN/T 3298-2012 "Determination of propylene units in ethylene-propylene copolymers for import and export - Infrared spectroscopy method" [9] and SH/T 1751-2005 "Determination of Ethylene in Ethylene-propylene copolymer (EPM) and ethylene-propylene-diene monomer (EPDM)"[10] developed by our laboratory. SN/T 3298 mainly targets at new materials, mainly blends with propylene as the main content, while SH/T 1751 mainly targets at ethylenepropylene rubber, which is inapplicable to the detection of recycled blends of polyethylene and polypropylene. Moreover, in daily tariff inspection, it is necessary to identify whether the polyethylene content in polyethylene and polypropylene blends is greater than $95 \%$, or whether the polypropylene content is greater than $95 \%$. The two different scenarios involve different HS codes and different tax rates. Therefore, it is necessary to develop a method for measuring the content of each component in the recycled polyethylene and polypropylene blends by infrared spectroscopy, which is particularly important for increasing our identification and quality control capabilities against imported plastic raw materials.

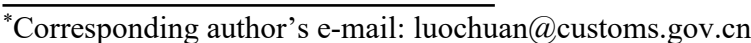




\section{Experimental part}

\subsection{Instruments and reagents}

Nexus Fourier Transform Infrared Spectrometer (with ATR accessory) (Thermo Fisher, U.S.), 35E injection molding machine (Boy, Germany), conventional laboratory tools.

Material: Linear Low Density Polyethylene (LLDPE) (Ningxia Baofeng Energy Co., Ltd., S1003), Low Density Polyethylene (LDPE) (Yanshan Petrochemical Corporation, 1C7A), High Density Polyethylene (HDPE) (Yanshan Petrochemical Corporation, 7600M), Polypropylene (Fujian United Petrochemical Company Limited, 2382E1).

Standard sample preparation: Weigh polyethylene and polypropylene: $98+2,96+4,95+5,96+4,92+8,90+10$, $75+25,50+50,25+75,10+90$, load them into a round bottom flask together, add sufficient toluene, and dissolve under heating and reflux. When there were no visible solid particles in the bottle, pour it out into a watch glass while it was hot, and place it in a ventilating hood for volatilization. After volatilization to near dryness, it was put into a blast oven and dried at $90^{\circ} \mathrm{C}$ to obtain standard samples of polyethylene and polypropylene blends of different compositions.

Experimental steps: Cut the sample, check whether the cut surface was flat and whether there were defects, ensure

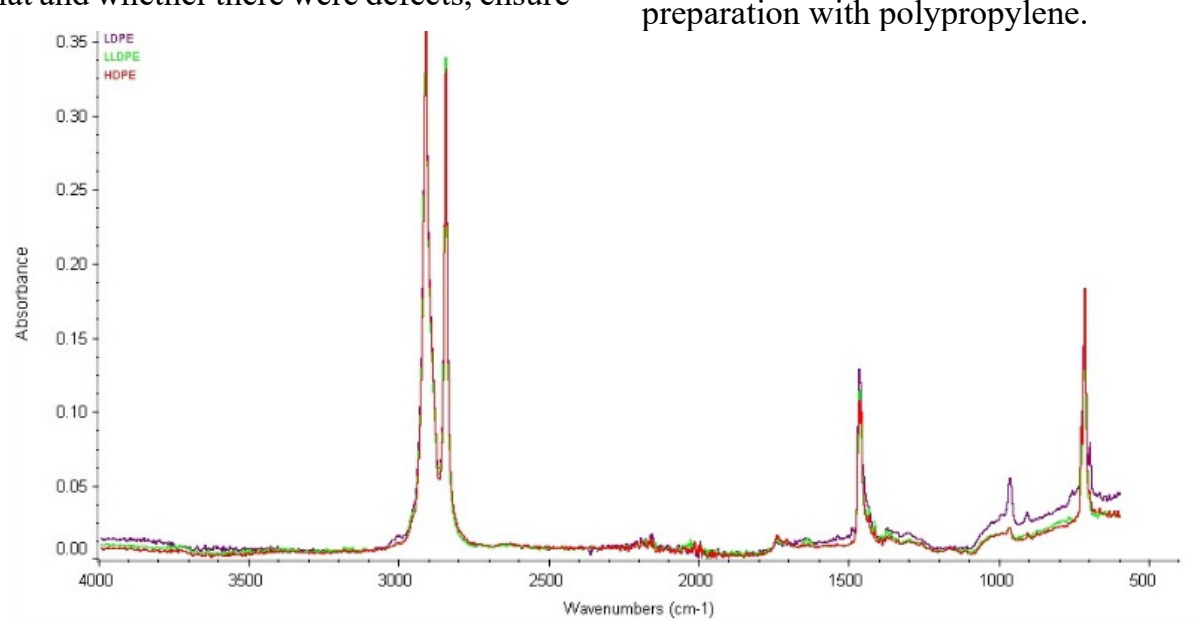

Figure 1 Standard spectrum diagram of low-density polyethylene, linear low-density polyethylene, and high-density polyethylene

\subsection{Selection of characteristic peaks}

Recycled plastics are different from conventional new materials. As recycled plastics, the sample will not only contain excessive oxidation products, but may contain many fillers and additives, especially carbon black that will seriously affect the infrared spectroscopy analysis. As shown in Figure 2, the blue represents recycled plastic, and the red represents virgin LLDPE. It can be seen that recycled plastic present a large number of miscellaneous peaks at $600 \mathrm{~cm}^{-1}-1400 \mathrm{~cm}^{-1}$. Therefore, it is quite necessary to select the appropriate characteristic peaks. According to SN/T 3298, the polypropylene has a characteristic peak at $1165 \mathrm{~cm}^{-1}$, polyethylene has a flat cut surface, put the sample under the infrared spectrum-ATR detector, and test after compaction: wave number: $400 \mathrm{~cm}^{-1}-4000 \mathrm{~cm}^{-1}$, scan times $>32$. Mark the positions of the characteristic peaks at $1165 \pm 5 \mathrm{~cm}^{-1}, 970 \pm 5$ $\mathrm{cm}^{-1}, 720 \pm 5 \mathrm{~cm}-1$ in the sample, and then calculate area and height of the characteristic peaks.

\section{Results and discussion}

\subsection{Selection of three kinds of PE}

Common polyethylene include low-density polyethylene (LDPE), linear low-density polyethylene (LLDPE), and high-density polyethylene (HDPE). The three types of polyethylene have differences in density and range of use, but with limited gap in infrared spectrum. New materials can be distinguished based on peak shape and absorbance of some characteristic peaks. However, in the detection of recycled plastics, it is difficult to distinguish the three kinds of polyethylene by infrared spectroscopy. From the superimposed spectrum of the three different PEs in Figure 1, it can be seen that the three kinds of PE have consistent main absorption peaks, so there is no need to establish three different PE/PP blend standard samples. Considering that LLDPE has intermediate melting point among the three kinds of PE, LLDPE was chosen as the standard polyethylene for solution blending-based sample preparation with polypropylene. characteristic peak at $720 \mathrm{~cm}^{-1}$. SH/T 1751 adopts the external standard method, and ethylene has characteristic peaks at $722 \mathrm{~cm}^{-1}, 1156 \mathrm{~cm}^{-1}, 1379 \mathrm{~cm}^{-1}, 1460 \mathrm{~cm}^{-1}$ etc. Referring to other literature, polypropylene has additional characteristic peak at $970 \mathrm{~cm}^{-1}$, and $970 \mathrm{~cm}^{-1}$ is for $\mathrm{CH}\left(\mathrm{CH}_{3}\right)$-characteristic peak. Seen from Figure 3, 1156 $\mathrm{cm}^{-1}$ is for the same characteristic peak as $1165 \mathrm{~cm}^{-1}, 1379$ $\mathrm{cm}^{-1}$ is mainly for bending vibration of methyl group, $1460 \mathrm{~cm}^{-1}$ is for bending vibration of $\mathrm{C}-\mathrm{H}$. These two characteristic peaks are more susceptible to the influence of additives such as silica and calcium carbonate, while $720 \mathrm{~cm}^{-1}$ is for the main resonance peak of $-\mathrm{CH}_{2}$-and the characteristic peak of polyethylene, so $720 \mathrm{~cm}^{-1}$ is selected as the characteristic peak of polyethylene, with $1165 \mathrm{~cm}^{-1}$ and $970 \mathrm{~cm}^{-1}$ as the characteristic peaks of polypropylene. 
The peak area of each characteristic peak and the actual sample content are listed in Table 1 below:

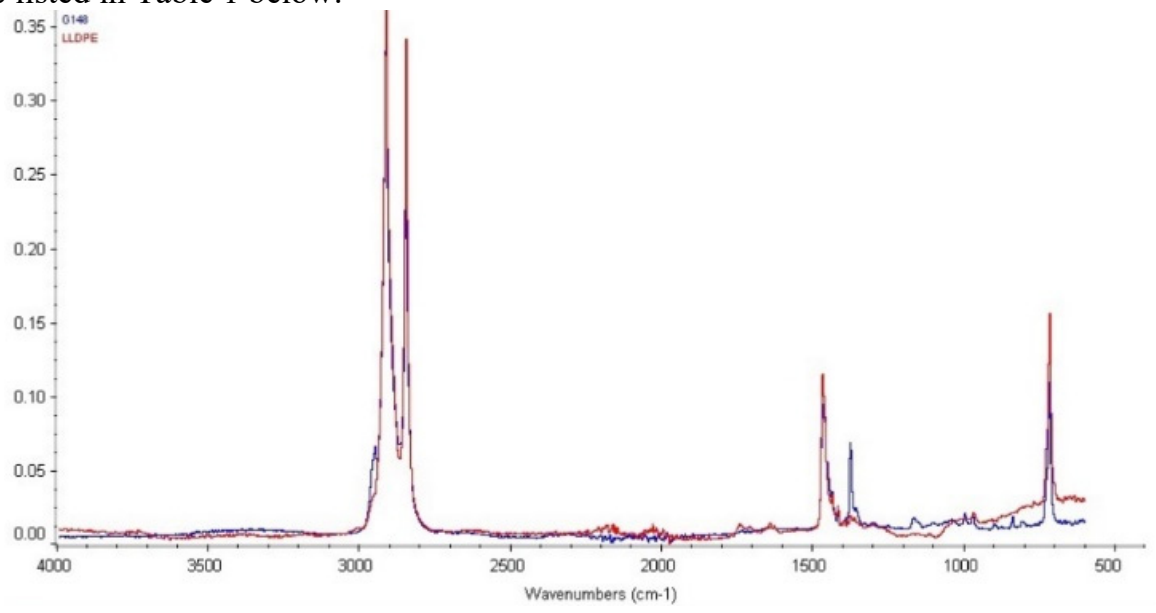

Figure 2 Diagram of polyethylene standard samples and recycled plastic samples

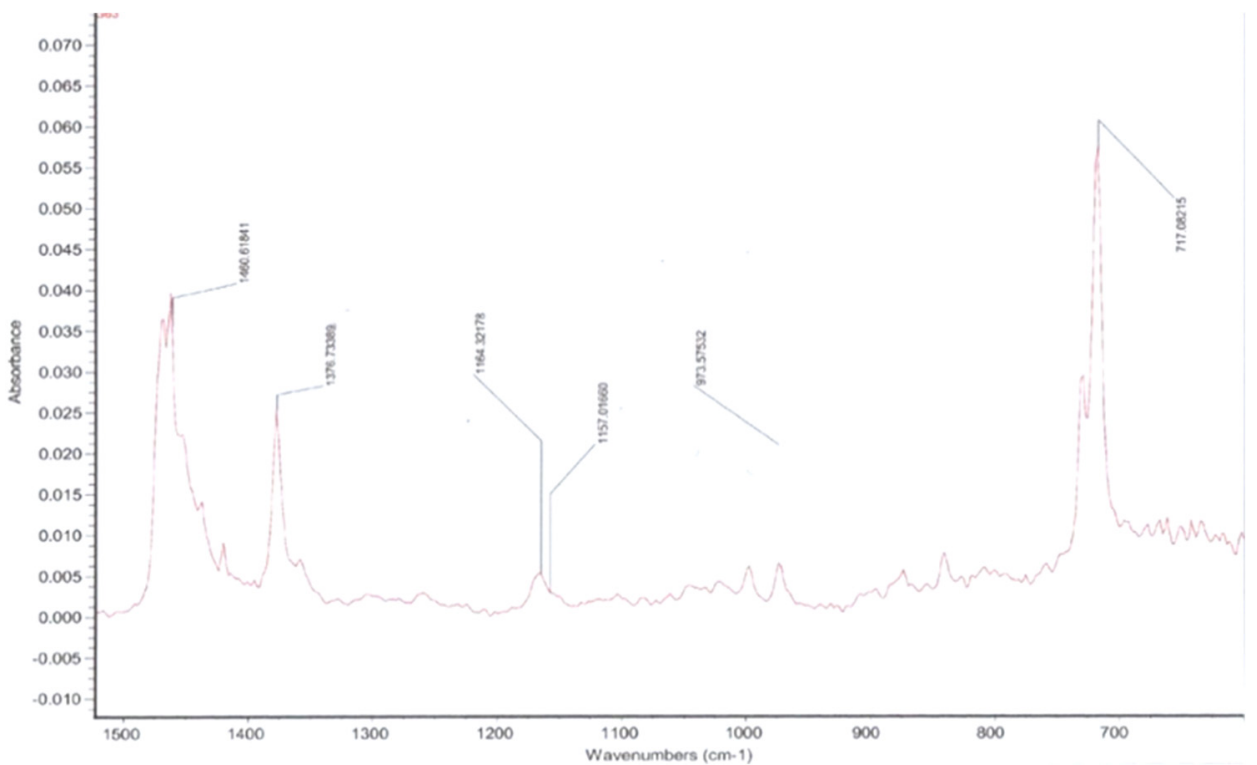

Figure 3 Characteristic peaks of polyethylene and polypropylene

The series standard samples were tested according to the above-mentioned test method, and the peak areas and peak heights of characteristic peaks were measured at 720 $\mathrm{cm}^{-1}, 970 \mathrm{~cm}^{-1}$ and $1165 \mathrm{~cm}^{-1}$, with the results shown in Table 1:

Table 1 The characteristic peak area and peak height of standard samples

\begin{tabular}{ccccccccc}
\hline No. & PE Cont. & PP Cont. & Area 1165 & Area970 & Area720 & $\begin{array}{c}\text { Peak } \\
\text { height } 1165\end{array}$ & $\begin{array}{c}\text { Peak } \\
\text { height } 970\end{array}$ & $\begin{array}{c}\text { Peak } \\
\text { height } 720\end{array}$ \\
\hline $98+2$ & $98.0 \%$ & $2.0 \%$ & 0.0341 & 0.0238 & 1.8722 & 0.0029 & 0.0029 & 0.1314 \\
$96+4$ & $96.0 \%$ & $4.0 \%$ & 0.0372 & 0.0302 & 1.7515 & 0.0041 & 0.0038 & 0.0832 \\
$95+5$ & $95.0 \%$ & $5.0 \%$ & 0.0367 & 0.0350 & 1.5157 & 0.0037 & 0.0035 & 0.0817 \\
$94+6$ & $94.0 \%$ & $6.0 \%$ & 0.0715 & 0.0652 & 1.7702 & 0.0056 & 0.0064 & 0.0785 \\
$92+8$ & $92.0 \%$ & $8.0 \%$ & 0.1245 & 0.0939 & 1.9848 & 0.0088 & 0.0094 & 0.1444 \\
$90+10$ & $90.0 \%$ & $10.0 \%$ & 0.1184 & 0.0986 & 1.7150 & 0.0052 & 0.0058 & 0.1290 \\
$75+25$ & $75.0 \%$ & $25.0 \%$ & 0.1507 & 0.1721 & 1.3033 & 0.0089 & 0.0096 & 0.0902 \\
$50+50$ & $50.0 \%$ & $50.0 \%$ & 0.2001 & 0.1600 & 1.0899 & 0.0127 & 0.0158 & 0.0782 \\
$25+75$ & $25.0 \%$ & $75.0 \%$ & 0.3136 & 0.3321 & 0.2082 & 0.0207 & 0.0299 & 0.0203 \\
$10+90$ & $10.0 \%$ & $90.0 \%$ & 0.2947 & 0.3269 & 0.1927 & 0.0193 & 0.0289 & 0.0105 \\
\hline
\end{tabular}




\subsection{Selection of peak area and peak height}

The peak area and peak height of the above-mentioned standard samples were compared and then calculated, with the results shown in Table 2:

Table 2 Comparison of peak area and peak height

\begin{tabular}{ccccccc}
\hline \multirow{2}{*}{ No. } & \multirow{2}{*}{ PE Cont. } & PP Cont. & \multicolumn{2}{c}{ Area ratio } & \multicolumn{2}{c}{ Peak heigh ratio } \\
\cline { 4 - 6 } & & & $1165 / 720$ & $970 / 720$ & $1165 / 720$ & $970 / 720$ \\
\hline $98+2$ & $98.0 \%$ & $2.0 \%$ & 0.0182 & 0.0127 & 0.0217 & 0.0221 \\
$96+4$ & $96.0 \%$ & $4.0 \%$ & 0.0212 & 0.0173 & 0.0492 & 0.0458 \\
$95+5$ & $95.0 \%$ & $5.0 \%$ & 0.0242 & 0.0231 & 0.0447 & 0.0430 \\
$94+6$ & $94.0 \%$ & $6.0 \%$ & 0.0404 & 0.0368 & 0.0718 & 0.0810 \\
$92+8$ & $92.0 \%$ & $8.0 \%$ & 0.0627 & 0.0473 & 0.0610 & 0.0650 \\
$90+10$ & $90.0 \%$ & $10.0 \%$ & 0.0690 & 0.0575 & 0.0403 & 0.0450 \\
$75+25$ & $75.0 \%$ & $25.0 \%$ & 0.1156 & 0.1320 & 0.0987 & 0.1064 \\
$50+50$ & $50.0 \%$ & $50.0 \%$ & 0.1836 & 0.1468 & 0.1624 & 0.2020 \\
$25+75$ & $25.0 \%$ & $75.0 \%$ & 1.5062 & 1.5951 & 1.0197 & 1.4729 \\
$10+90$ & $10.0 \%$ & $90.0 \%$ & 1.5293 & 1.6964 & 1.8381 & 2.7524 \\
\hline
\end{tabular}

Use the PP content of a standard sample with a PP content of $2 \%$ to $50 \%$ as the $\mathrm{Y}$-axis, and use $1165 / 720$ area ratio, 970/720 area ratio, $1165 / 720$ peak height ratio and

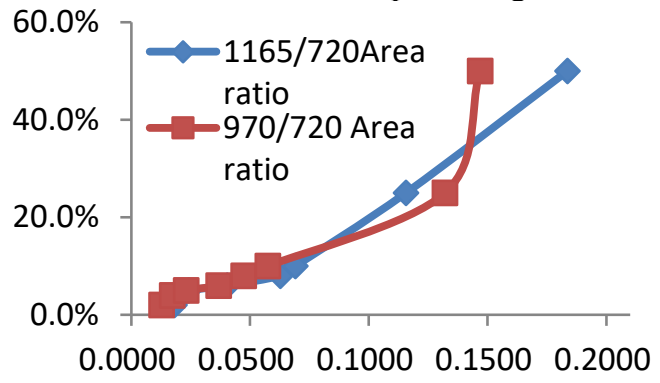

Figure $\square$ Relationship between PP content ratio and characteristic peak area ratio under $2 \%-50 \%$ PP content

It can be seen from Figure $\square$ and Figure $\square$ that when the area ratio of $1165 / 720$ and the area ratio of $970 / 720$ are used, the curve is close to the reverse S-shaped curve, which can be fitted in different ranges, while the curve with $1165 / 720$ peak height ratio and 970/720 peak height ratio is completely irregular, so peak height is not recommended for calculating PP content in the blend.
970/720 peak height ratio as the $\mathrm{X}$-axis for plotting, as shown in Figure $\square$ and Figure $\square$ :

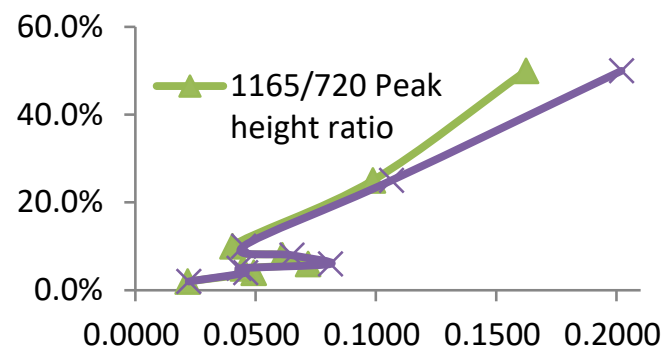

Figure $\square$ Relationship between PP content and characteristic peak height ratio under 2\%-50\% PP content

\subsection{Establishment of calibration curve}

As the curve presents a reverse S-shape, the PP content is divided into two intervals of $2 \%-8 \%$ and $8 \%-50 \%$ to establish calibration curves respectively, with the peak area of $1165 / 720$ and the peak area of 970/720 marked respectively. The results are shown in Figure $\square$ and Figure 7 


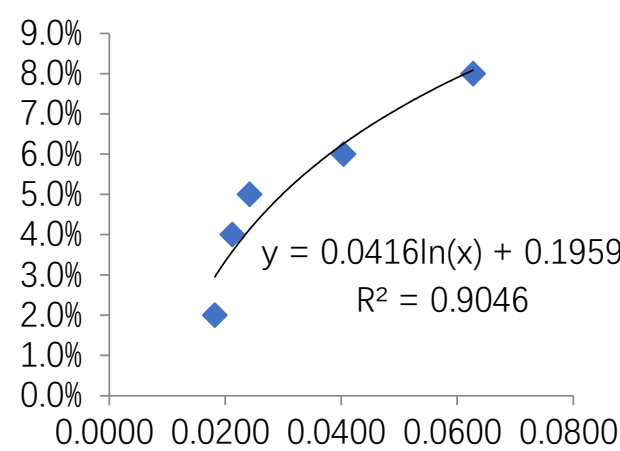

a) $1165 / 720$ peak area ratio

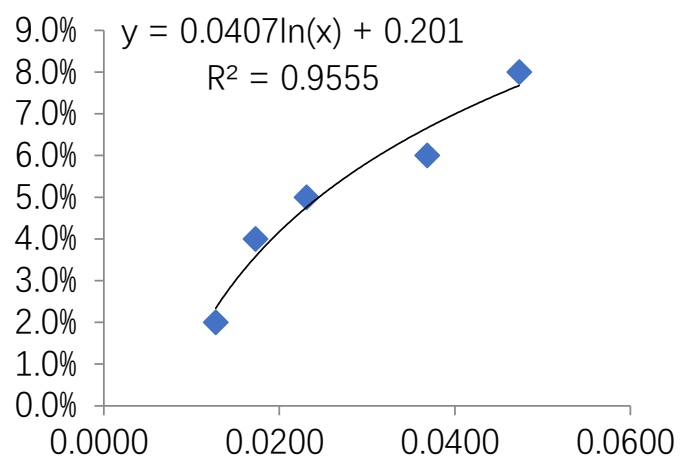

b) $970 / 720$ peak area ratio

Figure 6 The relationship between the characteristic peak area ratio and the content ratio under 2\% - $8 \%$ PP content

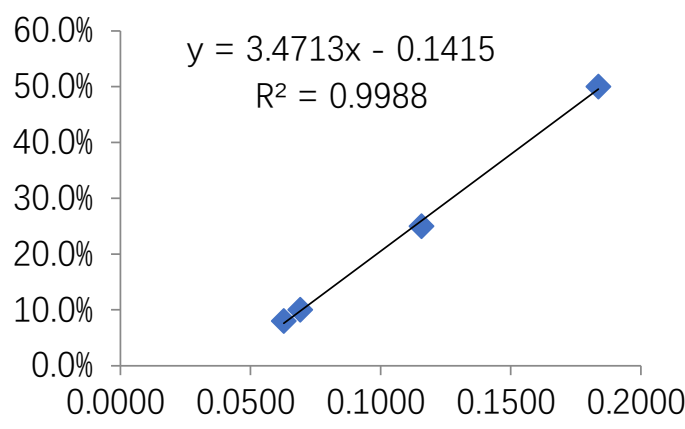

a) $1165 / 720$ peak area ratio

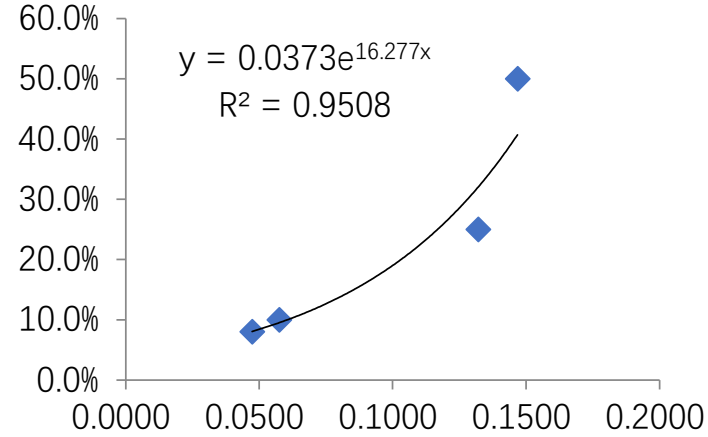

b) $970 / 720$ peak area ratio

Figure 7 The relationship between the characteristic peak area ratio and the content ratio under $8 \%-50 \%$ PP content

It can be seen from Figure 7 that the fitting of 970/720 peak area ratio and PP content presents a logarithmic fitting under $2 \%-8 \%$ PP content, and the correlation coefficient is greater than 0.95 , which is higher compared to $1165 / 720$ peak area ratio. Under $8 \%-50 \%$ PP content, $1165 / 720$ peak area ratio and PP content present a linear fitting, with a correlation coefficient greater than 0.995 , which is higher compared to $970 / 720$ peak area ratio, so PP content of recycled plastics is divided into two intervals for analysis:

When the content of PP is in the range of $2 \%$ to $8 \%$

Content of PP: $\mathrm{c}_{\mathrm{PP}}=0.0407 \ln \frac{\mathrm{A}_{970}}{\mathrm{~A}_{720}}+0.201$

When the PP content is in the range of $8 \%$ to $50 \%$

$$
\text { Content of PP: } \mathrm{C}_{\mathrm{PP}}=3.4713 \frac{\mathrm{A}_{1165}}{\mathrm{~A}_{720}}-0.1415
$$

Where:

A $_{970}: 970 \pm 5 \mathrm{~cm}^{-1}$ Characteristic peak area; $\mathrm{A}_{1165}: 1165 \pm 5 \mathrm{~cm}^{-1}$ Characteristic peak area; $\mathrm{A}_{720}: 720 \pm 5 \mathrm{~cm}^{-1}$ Characteristic peak area;

\subsection{Method accuracy}

Select $95+5$ standard sample, $90+10$ standard sample and $75+25$ standard sample for 7 consecutive tests, and calculate the PP content according to the above formula, with the results shown in Table 3 :

Table 3 Method accuracy

\begin{tabular}{|c|c|c|c|c|c|c|c|c|c|}
\hline $\begin{array}{c}\text { Standard } \\
\text { sample }\end{array}$ & 1 & 2 & 3 & 4 & 5 & 6 & 7 & AVG. & Max.deviation \\
\hline $95+5$ & $4.8 \%$ & $4.1 \%$ & $5.7 \%$ & $4.4 \%$ & $4.9 \%$ & $5.9 \%$ & $4.3 \%$ & $4.9 \%$ & $0.9 \%$ \\
\hline $90+10$ & $10.5 \%$ & $11.6 \%$ & $11.0 \%$ & $10.1 \%$ & $9.2 \%$ & $8.0 \%$ & $11.6 \%$ & $10.3 \%$ & $2.0 \%$ \\
\hline $75+25$ & $22.9 \%$ & $26.2 \%$ & $26.2 \%$ & $25.2 \%$ & $23.1 \%$ & $27.2 \%$ & $23.4 \%$ & $24.9 \%$ & $2.2 \%$ \\
\hline
\end{tabular}

It can be seen from Table 4 that the maximum absolute difference between the test result and the actual value of $95+5$ standard sample is $0.9 \%$, while the maximum absolute difference for $90+10$ standard sample and $75+25$ standard sample is $2.0 \%$ and $2.2 \%$, respectively.

\subsection{Method repeatability}

Select 4 samples with different proportions for 7 consecutive tests according to the above method, and calculate the repeatability of the method, with the results shown in Table 5: 
Table 4 Method repeatability

\begin{tabular}{llllrllllr}
\hline Sample & 1 & 2 & 3 & 4 & 5 & 6 & 7 & AVG. & \multicolumn{1}{c}{ RSD } \\
\hline GXT-2 & $7.1 \%$ & $6.5 \%$ & $9.2 \%$ & $10.4 \%$ & $7.4 \%$ & $8.1 \%$ & $7.3 \%$ & $8.0 \%$ & $17.1 \%$ \\
GXT-3 & $1.8 \%$ & $2.4 \%$ & $1.2 \%$ & $1.7 \%$ & $1.3 \%$ & $1.8 \%$ & $1.1 \%$ & $1.6 \%$ & $27.5 \%$ \\
GXT-4 & $6.8 \%$ & $6.0 \%$ & $6.1 \%$ & $7.5 \%$ & $6.8 \%$ & $6.3 \%$ & $7.7 \%$ & $6.7 \%$ & $9.8 \%$ \\
GXT-5 & $5.4 \%$ & $5.8 \%$ & $4.6 \%$ & $5.4 \%$ & $6.2 \%$ & $5.1 \%$ & $4.9 \%$ & $5.3 \%$ & $10.7 \%$ \\
\hline
\end{tabular}

It can be seen that when the polypropylene content is at the boundary, GXT-3 has relatively big relative standard deviation, while when the polypropylene content is about $5 \%$, the relative standard deviation of GTX-4 and GTX-5 is about $10 \%$, which can meet the basic test requirements.

\subsection{Actual sample detection and DSC method verification}

The differential scanning calorimetry results of 22 batches of actual PE/PP blends were selected for comparison with the infrared method results, as shown in Table 5:

Table 5 DSC method-based verification results

\begin{tabular}{cccccl}
\hline No. & A970/A720 & $\begin{array}{c}\text { content of PP test } \\
\text { by DSC }\end{array}$ & $\begin{array}{c}\text { content of PP test } \\
\text { by FTIR }\end{array}$ & Difference & Remark \\
\hline G118 & 0.0147 & $1.7 \%$ & $2.9 \%$ & $1.3 \%$ & Formula (1) calculation \\
G125 & 0.0150 & $2.3 \%$ & $3.0 \%$ & $0.7 \%$ & Formula (1) calculation \\
G65 & 0.0097 & $2.5 \%$ & $1.2 \%$ & $1.2 \%$ & Formula (1) calculation \\
G66 & 0.0107 & $2.5 \%$ & $1.6 \%$ & $0.8 \%$ & Formula (1) calculation \\
G155 & 0.0416 & $5.7 \%$ & $7.2 \%$ & $1.4 \%$ & Formula (1) calculation \\
G156 & 0.0283 & $6.1 \%$ & $5.6 \%$ & $0.5 \%$ & Formula (1) calculation \\
G151 & 0.0318 & $6.2 \%$ & $6.1 \%$ & $0.2 \%$ & Formula (1) calculation \\
G165 & 0.0501 & $6.4 \%$ & $7.9 \%$ & $1.5 \%$ & Formula (1) calculation \\
G104 & 0.0279 & $6.7 \%$ & $5.5 \%$ & $1.2 \%$ & Formula (1) calculation \\
G64 & 0.0462 & $10.0 \%$ & $1.9 \%$ & $8.1 \%$ & Formula (2) calculation \\
G57 & 0.0623 & $11.2 \%$ & $7.5 \%$ & $3.8 \%$ & Formula (2) calculation \\
F56 & 0.0580 & $16.1 \%$ & $6.0 \%$ & $10.1 \%$ & Formula (2) calculation \\
G127 & 0.0999 & $17.4 \%$ & $20.5 \%$ & $3.1 \%$ & Formula (2) calculation \\
G162 & 0.0432 & $19.0 \%$ & $0.9 \%$ & $18.2 \%$ & Formula (2) calculation \\
G67 & 0.0903 & $19.4 \%$ & $17.2 \%$ & $2.2 \%$ & Formula (2) calculation \\
G154 & 0.0873 & $24.3 \%$ & $16.2 \%$ & $8.1 \%$ & Formula (2) calculation \\
\hline
\end{tabular}

Seen from the results of actual samples, PP content between $2 \%$ and $8 \%$ as calculated by formula (1) has a good match with the melting area normalization method using DSC. The maximum difference is $1.6 \%$. At key value of 5\%, DSC results and FTIR results are completely consistent, both of which can well determine whether the polypropylene content in the polyethylene and polypropylene blend is less than $5 \%$. The difference between the PP content calculated by formula (2) and the DSC result is extremely uneven, ranging from $2.2 \%$ to $18.2 \%$. In the actual inspection process, the peak at 1165 $\mathrm{cm}-1$ has small peak shape due to addition of additives or fillers in the sample, so integration is uneasy, while peak at $970 \mathrm{~cm}-1$ always has a good peak shape and receives less interference.

Based on the above analysis, formula (2) cannot be used, only formula (1) has good precision and accuracy. By judging whether the A970/A720 ratio is greater than 0.0245 , it is possible to rapidly detect whether polypropylene content in polyethylene and polypropylene blends is greater than $5 \%$ in the tariff inspection.

\section{Conclusions}

This paper established a method for detecting polypropylene content in polyethylene and polypropylene blends using Fourier transform infrared spectroscopy. When polypropylene content was between $2 \%$ and $8 \%$, the ratio between polypropylene content and peak areas of the two characteristic peaked at $970 \mathrm{~cm}^{-1}$ and $720 \mathrm{~cm}^{-1}$ presented an exponential relationship. A correlation curve between the polypropylene content and the characteristic peak area has been established. The correlation coefficient was greater than 0.95 , and compared with differential scanning calorimetry, the results were basically consistent. This method can quickly determine whether the polypropylene content in the polyethylene and polypropylene blends is greater than $5 \%$ in the tariff inspection, and determine whether the polyethylene is mixed with a small amount of polypropylene content in the identification of plastic solid waste attributes.

\section{Acknowledgements}

This work was financially supported by the science research project of General Administration of Customs, P. 
R. CHINA (Fund No.: 2019HK016 and 2020HK245), the Ningbo public science research project (Fund No.: 2019C50031) and the Zhejiang basis public science research project (Fund No.: LGC20B040001).

\section{References}

1. DONG YM, XIONG XP, ZHENG W, etc., (2011) GAOFENZI YANJIU FANGFA. China Petrochemical Press Co. LTD, Beijing.: China

2. State Administration for Market Regulation and Standardization administration. (2019) General rules for infrared anaylsis: GB/T 6040-2019. Standards Press of China, Beijing.

3. YANG L, ZHOU ZC, YAO ZP, etc., (2019) Qualitative and quantitative analysis for PA6/PA66 compounds and co-polymers. CHINA SYNTHETIC RESIN AND PLASTICS. 36(2):7-9,18

4. ZHOU MY, YAN LK, JIAO GY. combined test metbcd with IR and DSC for plastics. Plastics SCI. \& Technology. 125(3):47-52

5. Luo C, Dai SJ, Zhang Y., etc. (2021) Identification Method of Solid Waste Characteristic for Imported Recycled Engineering Plastics. Earth Environ. Sci. 621(2021)012050

6. ZHANG Y, LUO C, YUAN LF, etc. (2021)
Pyrolysis-gas chromatography-mass spectrometry to identification of the solid waste characteristic of imported polyamide recycled plastics. Earth Environ. Sci. 621(2021)012038

7. YUAN LF, LUO C, XU SH, etc., (2020) Quantitative analysis of blend ratio of polycarbonate to acrylonitrile-butadiene-styrene copolymer by pyrolysis gas chromatography - mass spectrometry using solid dispersant for sample preparation, Journal of Instrumental Analysis 39(6):69-773

8. Luo C., Wang H., Wang Q., etc. (2020) Application of Instrumental in the Identification of Solid Waste Characteristic of Imported recycled ABS Plastic. Earth. Environ.Sci., 508(2020)012205

9. General Administration of Quality Supervision, Inspection and Quarantine of the People's Republic of China and Standardization Administration of the People's Republic of China. (2012) Determination of propylene units in ethylene-propylene copolymer for import and export - infrared spectroscopy method: SN/T 3298-2012. Standards Press of China, Beijing.

10. National Development and Reform Commission. (2005) Determination of ethylene units in ethylenepropylene copolymer (EPM) and ethylene-propylenediene terpolymers (EPDM): SH/T 1751-2005. China Petrochemical Press Co. LTD, Beijing. 